\title{
Analyse de la flore, de la végétation ligneuse et des fonctions des vallées en zone péri-urbaine post-conflit (Ziguinchor, Sénégal)
}

\author{
Maurice DASYLVA ${ }^{1 *}$, Ngor NDOUR $^{1}$, Ousmane NDIAYE $^{1}$ et Bienvenu SAMBOU ${ }^{2}$ \\ ${ }^{1}$ Département d'Agroforesterie, Laboratoire d'Agroforesterie et d'Ecologie (LAFE), Université Assane Séck de \\ Ziguinchor, BP 523 Ziguinchor, Sénégal. \\ ${ }^{2}$ Institut des Sciences de l'Environnement, Faculté des Sciences et Techniques, Université Cheikh Anta Diop de \\ Dakar, BP 5005 UCAD Dakar, Sénégal. \\ *Auteur correspondant ; E-mail : dasylvamaurice@gmail.com, Tél: + 221774538175.
}

\section{RESUME}

Cette étude contribue à une meilleure connaissance de la végétation ligneuse et des fonctions des vallées de Ziguinchor. 58 relevés de végétation, de $2500 \mathrm{~m}^{2}$ chacun, ont été réalisés et des enquêtes menées auprès de 153 ménages. La flore est riche de 90 espèces réparties dans 79 genres relevant de 35 familles. Les Fabaceae, les Anacardiaceae et les Moraceae plus représentées comptent respectivement $10 ; 7$ et 6 espèces chacune. L'indice de Jaccard $(0,53)$ apprécie la similarité de la flore des vallées de Diéfaye et Cobitène dominées, à $70 \%$, par Elaeis guineensis Jacq. La hauteur moyenne de peuplement $(8,88 \pm 0,57 \mathrm{~m})$ indique un faciès de végétation arborescent. L'indice de Shannon $(3,15)$ montre une diversité végétale relativement élevée dans ces vallées menacées par l'extension urbaine malgré leur fonction de production agricole selon $67,4 \%$ des ménages. Fort de ce constat, $74,5 \%$ des ménages plaident le maintien de leur fonction de production agricole. Cette doléance des populations, associée aux risques d'inondations des dépressions devraient amener les autorités municipales à promouvoir la sécurité alimentaire dans cette zone post-conflit par la préservation de la fonction de production agricole et de poumon vert des vallées.

(C) 2017 International Formulae Group. All rights reserved.

Mots clés : Vallées, végétation, flore, ménage, production agricole.

\section{Analysis of the flora, the woody vegetation and the functions of the valleys in peri-urban post-conflict zone (Ziguinchor, Senegal)}

\begin{abstract}
This study contributes to a better understanding of the woody vegetation and functions of Ziguinchor's valleys. 58 vegetation inventory units of $2500 \mathrm{~m}^{2}$ each were delimited in Cobitene and Diefaye valleys and survey were carried out on 153 households. The flora is rich of 90 species distributed in 79 genera and 35 families. The Fabaceae, Anacardiaceae and Moraceae are more represented with respectively 10; 7 and 6 species. The index of Jaccard (0.53) appreciates the similarity of the flora of Diéfaye and Cobitène valleys dominated to $70 \%$, by Elaeis guineensis Jacq. The average height of population $(8.88 \pm 0.57 \mathrm{~m})$ indicates an arborescent facies of vegetation. The index of Shannon (3.15) shows a vegetal diversity relatively high in these valleys threatened by the urban extension in spite of their function of agricultural production according to
\end{abstract}


$67.4 \%$ of the households. Based on this result, $74.5 \%$ of the households plead the maintenance of their function of agricultural production. This complaint of the populations, associated with the risks of floods in the depressions, should lead municipal authorities to promote food safety in this post-conflict zone by safeguarding valley's agricultural functions.

(C) 2017 International Formulae Group. All rights reserved.

Keywords: Valleys, vegetation, flora, household, agricultural production.

\section{INTRODUCTION}

Dans les villes en voie de développement, les terres agricoles sont occupées par le bâti en raison d'une croissance démographique exponentielle (Oladokoun, 2013). Selon Boucher et Fontaine (2010), l'urbanisation galopante et ses corollaires expliquent la perte d'espèces végétales et de leurs services écosystémiques dans les villes. Pourtant, les territoires urbains comportent de forts potentiels en termes de diversité végétale et présentent de nombreuses possibilités de valorisation (Diané, 2000; Dasylva, 2013). Dans le contexte actuel de la communalisation au Sénégal; la particularité de Ziguinchor, réside dans son urbanisation galopante au détriment des terres agricoles et leur diversité floristique. En effet, la région de Ziguinchor est l'une des plus urbanisée du Sénégal avec un taux évalué à $46 \%$ (ANSD, 2014). La ville régionale concentre l'essentielle de la population des centres urbains de son département (ANSD, 2015). D'ailleurs, ce phénomène d'urbanisation de la ville a été exacerbé par trente ans de crise sociale de la Casamance (Dasylva, 2013). Cet état des faits se traduit par l'extension de la ville et l'occupation anarchique et progressive des plaines et vallées inondables. Non aedificandi, ces zones inondables sont destinées à la production agricole et constituent l'espace vert de la ville (Mbaye et al., 2011; Dasylva, 2013). L'occupation de ces espaces entraine la dégradation de la végétation ligneuse et de ses divers services écosystémiques que les citadins en tirent. A cet égard, si rien n'est fait à l'avenir, les travaux de Dasylva (2013) et Keita (2013) présagent d'une perte potentielle de plus 123 ha des terres agricoles à Ziguinchor. Malheureusement, les autorités municipales n'accordent, aux vallées rizicoles et maraichères, que la fonction de réserve foncière vouée à l'expansion de la ville alors que les producteurs dépendent exclusivement de ces vallées pour atténuer l'insécurité alimentaire en zone de conflit (PAM, 2012; Dasylva, 2013). Cette étude se propose de faire: une caractérisation de la végétation ligneuse et une analyse des fonctions environnementales et socioéconomiques des vallées de Ziguinchor.

\section{MATERIEL ET METHODES}

\section{Site de l'étude}

Cette étude a été réalisée à Ziguinchor; ville créée en 1888 et chef-lieu administratif de la région du même nom. Située au SudOuest du Sénégal, la commune est localisée entre le méridien $16^{\circ}$ et $17^{\circ}$ et les parallèles $12^{\circ}$ et $13^{\circ}$ (Figure 1 ). Cette ville, limitée au Nord par le fleuve Casamance, au Sud, à l'Est et à l'Ouest par la commune de Niaguis, est caractérisée par un climat de type soudanien côtier Sud (Sagna, 2005) ; et, une température et une pluviométrie moyennes annuelles respectivement évaluées, entre 1984 et 2015, à $27,10^{\circ} \mathrm{C}$ et $1322,66 \mathrm{~mm}$. Malgré la variabilité pluviométrique, Ziguinchor est l'une des villes les plus arrosées du Sénégal. Cette bonne pluviométrie corrélée à l'étendue des dépressions offre à cette ville un grand potentiel agronomique. Le climat se caractérise par une longue saison sèche qui 
dure environ sept à huit mois (novembre-mai) et une courte saison des pluies, de mai à octobre.

Selon l'ANDS (2015), Ziguinchor comptait 297172 habitants en 2013, répartis dans ses 27 quartiers administratifs. Sa position géographique, entre la Guinée-Bissau et la Gambie, lui confère une importante diversité culturelle et cultuelle qu'incarnent les ethnies dominantes notamment le Diola, le Mandingue, le Peulh, le Wolof, le Manjack, le Ballante, le Sérère et le Mancagne. Les cultures sous pluie et de contre saison, pratiquées dans les vallées des quartiers périurbains, demeurent un axe stratégique de développement locale et de lutte contre l'insécurité alimentaire des populations en zone post-conflit.

\section{Choix du site}

Le choix des sites a été réalisé sur la base de missions de reconnaissance ayant permis de recenser 5 vallées dans la ville. Il s'agit des vallées de Diéfaye, Cobitène, Lyndiane, Kenya et celle de Kandialang. Il a porté sur les vallées de Diéfaye et Cobitène qui focalisent l'essentiel de l'agriculture urbaine de la ville.

\section{Méthodes de collecte des données}

Les relevés de végétation ont été réalisés dans ces vallées, sur la base d'un taux de sondage de $3 \%$ de la superficie de chacune. Des placettes carrées de $50 \mathrm{~m}$ de côté $(0,25$ ha), ont été installées de façon systématique sur la base de transects. La méthode du 3-4-5 (three-four-five) à l'aide de 5 jalons, d'une ficelle de $50 \mathrm{~m}$ et d'un ruban métrique de 20 $\mathrm{m}$ a été utilisée pour la matérialisation des placettes. L'équidistance entre les placettes et entre les transects est de $20 \mathrm{~m}$. Les ligneux présents dans les placettes ont été notés. Les mensurations ont porté sur le diamètre à hauteur de poitrine, le diamètre croisé du houppier et la hauteur sur pied de chaque sujet adulte; respectivement à l'aide d'un compas forestier, d'un ruban mètre et d'un Blumeleiss. Les sujets de diamètre à hauteur de poitrine $(1,30 \mathrm{~m})$ inférieur à $7 \mathrm{~cm}$ et les palmiers de hauteur inférieure à $1,30 \mathrm{~m}$ (Diédhiou et al., 2014) sont considérés comme de la régénération naturelle. Dans chaque placette, la régénération naturelle, les souches et les individus morts sont comptés. Les observations ont permis de noter les activités humaines sur les ligneux (sujets élagués, écorcés, coupés, brûlés, etc.). Les ligneux non identifiés sur le terrain ont fait l'objet de prélèvement d'échantillons pour une identification au laboratoire à l'aide de clés de détermination notamment la «flore du Sénégal» de Berhaut (1967), certains ouvrages comme les «ligneux du Sahel» de Bonnet et al. (2005) et les « Arbres, arbustes et lianes des zones sèches de l'Afrique de l'Ouest » de Arbonnier (2009). Au total, 18 relevés de végétation ont été réalisés dans la vallée de Diéfaye, soit 1,24 km de transect et 40 autres dans la vallée de Cobitène soit, 2,78 $\mathrm{km}$ de transect.

Les enquêtes ont porté sur les usages des peuplements végétaux et les fonctions des vallées de la ville de Ziguinchor. Un questionnaire a été administré à 153 ménages répartis dans les quartiers périurbains de Diéfaye, Cobitène, Colobane, Lyndiane et Kandialang. Le choix a porté sur ces quartiers en raison de leur proximité avec les vallées et de la forte concentration des exploitants dans ces vallées. Les enquêtes ont été réalisées par la méthode du porte-à-porte dans ces quartiers.

\section{Traitement des données}

Le traitement des données de la vegetation a permis de déterminer, la composition floristique, la richesse spécifique moyenne et celle totale. Les données d'abondance dominance notées lors des relevées de végétation ont été traitées à l'aide 
$\mathrm{du}$ tableur Excel et du logiciel XLSTAT Pro 7.5.2 qui ont servi à l'élaboration des tableaux et des graphiques. Ces données ont été soumises à des Analyses en Composantes Principales (ACP) associées aux Test de comparaison de Fisher et celui de sphéricité de Bartlett au seuil de signification Alpha $=0,05$.

Utilisé en statistique pour comparer la différence entre des échantillons, l'indice de Jaccard a permis de déterminer la dissimilarité, en termes de diversité spécifique, entre les vallées de Cobitène et Diéfaye. Il est calculé par la relation: $D_{\mathrm{j}}\left(\mathrm{j}_{1}\right.$, $\left.\mathrm{j}_{2}\right)=1-[\mathrm{C} /(\mathrm{A}+\mathrm{B}+\mathrm{C})]$, où $(\mathrm{C})$ représente le nombre d'espèces communes aux deux sites $\mathrm{J}_{1}$ et $J_{2}$; (A) le nombre d'espèces propres au site $\mathrm{J}_{1}$ et (B) le nombre d'espèces propres au site $\mathrm{J}_{2}$. L'indice de Jaccard varie de 0 à 1 . La nullité de la valeur de l'indice indique que les deux sites sont de compositions floristiques identiques alors que la valeur maximale (1) montre que les deux relevés n'ont aucune espèce en commun. L'indice de diversité de Shannon-Weaver est basé sur la relation : $\mathrm{H}^{\prime}=$ - $\Sigma$ pi $\log _{2}$ pi, où pi est la fréquence de l'espèce i. H' est minimal (égal à 0 ) si tous les individus du peuplement appartiennent à une seule et même espèce et est maximal $(4,5)$ quand tous les individus sont répartis d'une façon égale (Frontier et Pichod-Viale, 1995). Cet indice calcule la diversité des groupes comparativement à la diversité maximale $\left(\mathrm{H}_{\max }\right)$ qui est le logarithme à base $2 \mathrm{du}$ nombre d'espèces (S); $\mathrm{H}_{\max }=\log _{2}(\mathrm{~S})$. L'indice d'équitable R de Piélou (1966), également appelé indice de régularité (Blondel, 1979 cité par Faye 2005) est déterminé par la relation : $\mathrm{R}=\mathrm{H}^{\prime} / \mathrm{H}_{\max }$. Il correspond au degré de réalisation de la diversité maximale (Faye, 2005). La densité du peuplement correspond au nombre d'individus par unité de surface et s'exprime en nombre d'individu par ha (ind/ha). Elle est obtenue par la relation : $\mathrm{D}=$ N/S. N représente l'effectif total d'individus dans l'échantillon considéré et $\mathrm{S}$ la surface de l'échantillon en ha. Le couvert ligneux désigne la surface de la couronne de l'arbre projetée verticalement au sol et est calculé par la relation: $\mathrm{C}=\sum\left[\left(\pi\left(\mathrm{d}_{\mathrm{mh}} / 2\right)^{2}\right) / \mathrm{S}_{\mathrm{E}}\right]$. La surface terrière correspond à la surface de l'arbre évalué à la base de son tronc et est obtenue par la relation $\mathrm{S}_{\mathrm{t}}=\sum\left[\left(\pi\left(\mathrm{d}_{1.3} / 2\right)^{2}\right) /\right.$ $\mathrm{S}_{\mathrm{E}}$ ]. Ces deux variables dendrométrique sont exprimés en mètre carré par hectare $\left(\mathrm{m}^{2} \cdot \mathrm{ha}^{-1}\right)$. $\mathrm{S}_{\mathrm{E}}$ correspond à la surface de l'échantillon considéré en ha, $\mathrm{d}_{\mathrm{mh}}$ au diamètre moyen $\mathrm{du}$ houppier et $\mathrm{d}_{1.3}$ au diamètre du tronc à $1,3 \mathrm{~m}$.

$\mathrm{La}$ régénération naturelle $\mathrm{du}$ peuplement permet de comprendre la dynamique de la végétation ligneuse. Le taux de régénération est déterminé par le rapport, en $\%$, entre l'effectif des jeunes plants et l'effectif total du peuplement (Poupon, 1980). L'Importance Spécifique de Régénération (ISR) est obtenu à partir du rapport, en \%, entre l'effectif de jeunes plants d'une espèce donnée et l'effectif total des jeunes plants dénombrés (Akpo \& Grouzis, 1996). Dans cette étude, l'ISR de l'espèce dominante du peuplement a été calculé. Le taux d'anthropisation est le rapport en \% de l'effectif des individus marqués par l'empreinte d'une activité humaine sur l'effectif total du peuplement.

Les données issues des enquêtes ont été traitées avec le logiciel Sphinx Plus². Ce logiciel a permis de réaliser des analyses univariées et multivariées; et, de déterminer les tendances centrales des variables liées aux perceptions des populations sur les services écosystémiques de la végétation ligneuse, des fonctions assignées aux vallées et le devenir de l'agriculture en milieu urbain à Ziguinchor. 


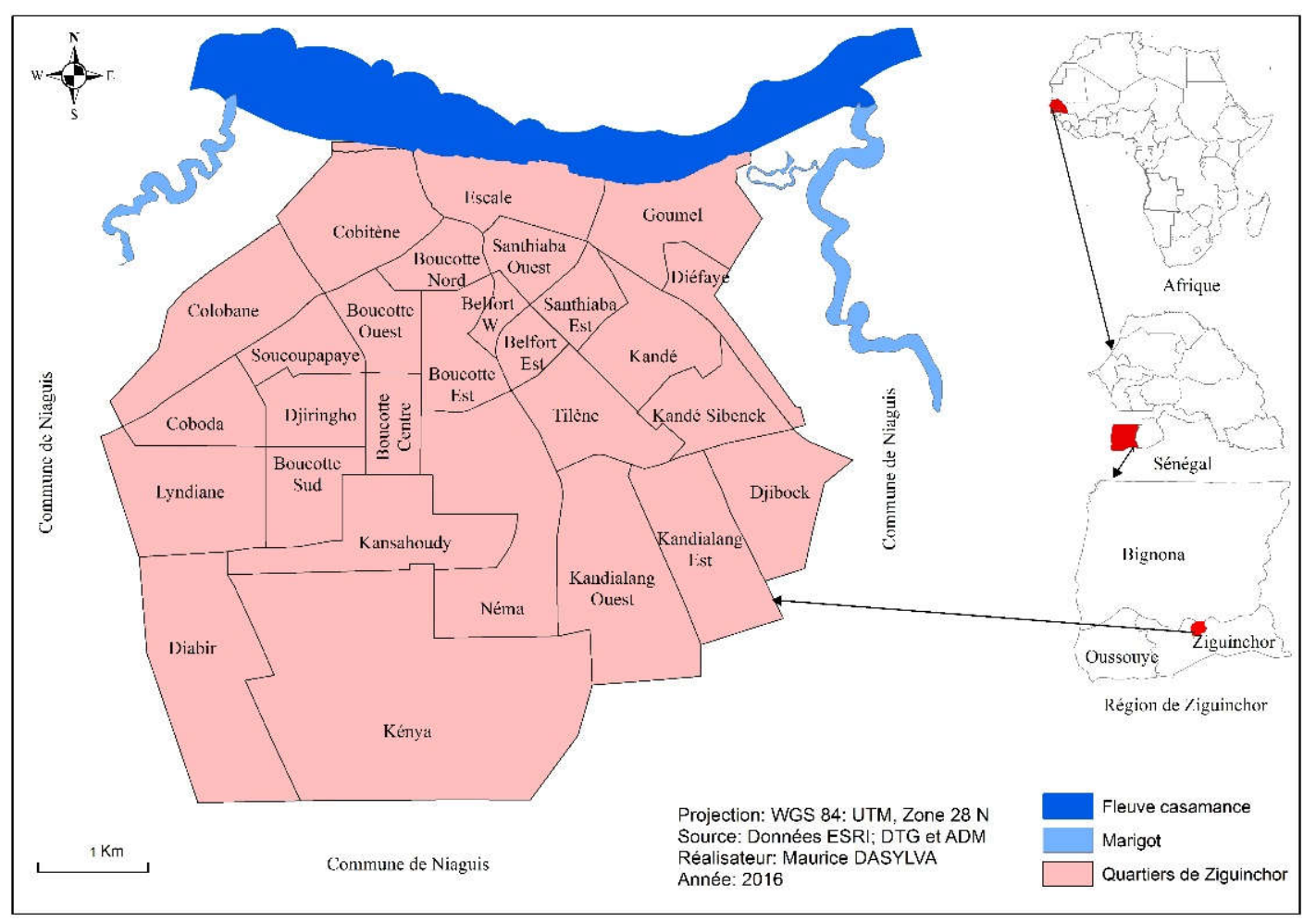

Figure 1 : Carte de localisation de la ville de Ziguinchor.

\section{RESULTATS}

\section{Richesse spécifique}

La richesse spécifique totale de la flore ligneuse dans les deux vallées est de 90 espèces. Elle est de 68 espèces dans la vallée de Diéfaye et 67 espèces dans celle de Cobitène (Tableau 1). La variation de la richesse spécifique moyenne par placette, entre les deux vallées, n'est pas significative $(p=0,13)$. Elle est, toutefois, plus importante dans la vallée de Diéfaye $(10,94 \pm 3,82$ espèces/placette) que dans celle de Cobitène $(6,88 \pm 0,97$ espèces/placette). La variance de la richesse spécifique moyenne qui permet de déterminer l'hétérogénéité d'un peuplement est plus élevée à Diéfaye $(15,16)$ qu'à Cobitène $(8,25)$. Elle montre ainsi que le peuplement de Cobitène est plus homogène que celui de Diéfaye en termes de distribution des espèces dans l'espace.

\section{Diversité spécifique et structure de la végétation}

Le Tableau 2 présente la variation de l'indice de diversité de Shannon dans les deux vallées. Il en ressort que l'indice de diversité de Shannon (3,15 bits) est relativement élevé à l'échelle des deux vallées. Il est cependant plus important dans la vallée de Cobitène que celle de Diéfaye. Par contre, la diversité maximale est plus importante à Diéfaye $(6,09$ $\pm 0,30)$ qu'à Cobitène. Cependant, le degré de réalisation de la diversité maximale est plus élevé à Cobitène qu'à Diéfaye. Il montre ainsi que le peuplement de Cobitène a une répartition de l'abondance des espèces plus équitable que celui de Diéfaye.

Le cortège floristique à l'échelle des deux vallées compte 90 espèces réparties dans 79 genres et 35 familles botaniques (Figure 2). La famille des Fabaceae, composée de Afzelia 
africana Sm., Cassia siamea, Cassia sieberiana DC.Lam., Detarium senegalense Gmel., Dialium guineense Willd., Delonix regia (Boj.) Raf., Piliostigma thonningii (Sch.) Miln. Redh, Senna alata (L.)Roxb. Erythrina senegalensis DC. et de Gliricidia sepium (Jacq.) Walp, est la plus représentée. Elle est suivie de la famille des Anacardiaceae constituée de Anacardium occidentale L., Anthostama senegalense A. Juss., Lannea acida A.Rich, Mangifera indica L., Sclerocarya birrea (A. Rich.), Spondias mombin $\mathrm{L}$. et de Thevetia neriifolia Juss. La famille des Mimosaceae et des Moraceae sont constituées de 6 espèces chacune alors que les Apocinaceae et les Euphorbiaceae comptent 5 espèces chacune. La majorité des familles $(54,29 \%)$ sont composées d'une seule espèce chacune. A l'exception des taxons de la famille des Arecaceae, 95,55\% des espèces appartiennent au groupe des monocotylédones. Le genre Ficus, composé de 6 espèces, est le plus représenté. Il est suivi des genres Citrus et Combretum qui sont constitués de 3 espèces chacune. Le genre Albizia est composé de 2 espèces. Les 93,67\% des genres ne comptent qu'une seule espèce chacune.

L'indice de dissimilarité de Jaccard, déterminé entre les deux vallées, est de 0,53. En effet, $51,11 \%$ des espèces sont communes aux deux vallées; $25,56 \%$ sont propres à la vallée de Diéfaye et $23,33 \%$ à celle de Cobitène. Il est à noter la présence de certaines espèces ligneuses typiques des basfonds comme Rhizophora mangle L., Mitragyna inermis (Willd.) Kuntze et Elaeis guineensis dans une moindre mesure.

\section{Analyse fréquentielle}

L'analyse fréquentielle établie à l'aide du tableau de présence/absence a permis de quantifier le cortège floral à l'échelle des deux vallées en se référant de la grille d'analyse des indices de Caratani (Tableau 3). Il ressort de ce tableau que 48 espèces de la vallée de Diéfaye et 62 espèces de celle de Cobitène ont une fréquence de présence inférieure à 0,2 ; soit, respectivement, $70,59 \%$ et $92,54 \%$ de la richesse spécifique totale. Elles sont dites rares. Quatre espèces (Combretum paniculatum Vent., Voacanga africana Stapf., Albizia adianthifolia (Sch.) et Alchornea cordifolia (S. et Th.) dans la vallée de Diéfaye et une espèce (Azadirachta indica A.Juss.) dans celle de Cobitène ont une fréquence de présence comprise entre 0,4 et 0,6 . Elles sont dites fréquentes. Les espèces dites abondantes, qui ont une fréquence de présence comprise entre 0,6 et 0,8 sont Azadirachta indica et Ficus vogelii Miq. dans la vallée de Diéfaye ; et Elaeis guineensis dans la vallée de Cobitène. Dans la vallée de Diéfaye, $E$. guineensis Jacq. présente une fréquence de présence de 0,9. Elle y est attribuée l'indice V (constante), traduisant sa présence dans tous les relevés de végétation de cette station.

Le Test de Student réalisé pour comparer la moyenne des variables des peuplements des vallées (Tableau 4), montre que leur différence n'est pas significative avec une probabilité de risque de $40 \%$. Ce tableau montre que le taux de régénération naturelle est relativement élevé à l'échelle des deux vallées et est estimé à $61,14 \pm 5,41 \%$. Il est plus important à Diéfaye $(63,31 \pm 10,90 \%)$ qu'a Cobitène $(60,01 \pm 6,90 \%)$. L'importance spécifique de régénération de $E$. guineensis est évalué à 33,30 $\pm 7,16 \%$ à l'échelle des deux vallées. Elle est plus élevée à Diéfaye et représente environ 2 fois celle de Cobitène évaluée à $23,69 \pm 8,72 \%$. Le taux d'anthropisation, par contre, est plus important à Cobitène $(35,84 \pm 7,08 \%)$ qu'à Diéfaye où il est évalué à $33,23 \pm 10,73 \%$. Le taux de mortalité estimé à l'échelle des deux vallées à $5,39 \pm 1,93 \%$ est plus important à Cobitène $(6,13 \pm 3,04 \%)$ qui constitue presque le double de celui déterminé à Diéfaye $(3,94$. $\pm 1,19 \%)$. 
Le Test de sphéricité de Bartlett a permis de montrer, au seuil de signification alpha $(0,05)$ qu'il existe une corrélation significative entre les indices de diversité de la flore, les taux de régénération, d'anthropisation et de mortalité du peuplement avec une probabilité de risque inférieure à $0,01 \%$. La richesse spécifique moyenne est positivement corrélé à $\mathrm{H}^{\prime}$ et $\mathrm{H}_{\max }$ et est négativement corrélé au taux d'anthropisation. Leurs coefficient de corrélation sont respectivement évalués à 0,$899 ; 0,571$ et $-0,292$. Par ailleurs, le taux d'anthropisation est négativement corrélé au taux de régénération naturelle et à $\mathrm{H}^{\prime}$ avec les degrés de corrélation respectifs de $-0,616$ et 0,356 .

La Figure 3 correspond à la projection vectorielle des variables du Tableau 6 et les relevés de végétation dans un système d'axes. Son examen montre que les facteurs (taux de régénération naturelle, richesse spécifique moyenne et $\mathrm{H}_{\max }$ sont positivement corrélés à l'axe 1 et aux relevés $1 ; 15 ; 17$ et 18 de Diéfaye et ceux 1 et 5 de Cobitène. Ces six (6) relevés concentrent l'essentiel de l'information qui justifie ladite corrélation. Par contre, les taux de mortalité et d'anthropisation sont négativement plus corrélés à l'axe 1 et aux relevés $3 ; 8 ; 19$; et 28 de Cobitène et ceux $3 ; 7$ et 10 de Diéfaye. Ces relevés sont beaucoup plus caractérisés par l'anthropisation et la mortalité de la végétation. Par ailleurs, l'indice spécifique de régénération de $E$. guineensis est négativement lié à l'axe 2 et au relevés $11 ; 12$ et 14 de Diéfaye et ceux 2 et 12 de Cobitène. Ces cinq (5) relevés concentrent l'essentiel de la régénération naturel du palmier à huile dans ces vallées.

Les paramètres structuraux de forme de la végétation sont essentiellement liés, dans le cadre de cette étude, à la densité observée, à la hauteur moyenne, au couvert ligneux et à la surface terrière du peuplement (Tableau 5). Le
Test $\mathrm{t}$ de Student effectué pour comparer la moyenne de ces variables, entre les vallées de Cobitène et Diéfaye, montre que leur différence n'est pas significative avec une probabilité de risque $19,1 \%$ au seuil alpha $(0,05)$. L'analyse de ce tableau montre que la végétation ligneuse est relativement dense dans les vallées de Ziguinchor. La densité observée totale du peuplement est globalement évaluée à $216,62 \pm 63,84$ pieds.ha ${ }^{-1}$. Cependant, elle est moins importante à Cobitène et représente, environ $1 / 3$ de celle déterminée à Diéfaye $(393,78 \pm$ 148,09 pieds.ha ${ }^{-1}$ ). La densité observée de $E$. guineensis représente globalement $55,14 \%$ de celle totale. Elle est plus importante à Diéfaye et représente environ 5 fois celle estimée à $53,40 \pm 15,89$ pieds.ha ${ }^{-1}$ à Cobitène. La hauteur moyenne du peuplement est estimée à $8,88 \pm 0,57 \mathrm{~m}$ à l'échelle des deux vallées. Le couvert ligneux mesure 1976,08 $\pm 1118,80$ $\mathrm{m}^{2} \cdot \mathrm{ha}^{-1}$ à Diéfaye et $862,59 \pm 270,27 \mathrm{~m}^{2} \cdot \mathrm{ha}^{-1}$ à Cobitène. La hauteur moyenne du palmier à huile est de 7,60 $\pm 0,67 \mathrm{~m}$. Elle est plus importante à Diéfaye $(9,75 \pm 0,52 \mathrm{~m})$ qu'à Cobitène $(6,64 \pm 0,79 \mathrm{~m})$. La distribution verticale de ses individus (Figure 4), est de type unimodal et est caractérisée par la présence de toutes les classes de hauteur. L'analyse de cette figure montre que dans la vallée de Cobitène, les classes de hauteur [6; $8[;[8 ; 10[;[4 ; 6[$ et $[10,12[$ sont les plus représentées avec une densité respective 13,5; 9,$0 ; 7,8 ; 7,3$ et 4,5 pieds.ha- ${ }^{1}$. Par contre, dans la vallée de Diéfaye, les classes de hauteur $[10,12[,[6 ; 8[;[8 ; 10[$ et $[0 ; 2[$ sont les plus représentées avec une densité respective de 21,$11 ; 14,44 ; 12,89$ et 8,75 pieds.ha- ${ }^{1}$. Environ $63,29 \%$ des individus de E. guineensis sont principalement concentrés dans les classes de hauteurs comprises entre 8 et 12 mètre dans les vallées. Toutefois, les classes de hauteur des grands sujets sont les moins représentées avec une densité ne 
dépassant pas 3 pieds/ha. Le Coefficient d'asymétrie, des courbes de tendance, de Pearson qui permet de comparer l'homogénéité de distribution des individus d'un peuplement est évalué à 0,669 à Cobitène et 1,189 à Diéfaye. Ils permettent de déduire que la structure verticale de la palmeraie de Cobitène est plus homogène que celle de Diéfaye.

Le Test de sphéricité de Bartlett a permis de noter, au seuil de signification alpha $(0,05)$, une corrélation significative entre richesse spécifique moyenne et les paramètres de forme du peuplement avec une probabilité de risque de $0,01 \%$. La corrélation est plus significative entre la richesse spécifique moyenne et la densité observée totale, d'une part, et, d'autre part, entre la richesse spécifique moyenne et le couvert ligneux. Leurs coefficients de corrélation respectifs sont 0,788 et 0,602 . Le couvert ligneux et la densité observée sont aussi corrélés à $65,6 \%$. La Figure 5 présente la corrélation entre les paramètres de formes du peuplement et les relevés floristiques. L'analyse de cette figure permet de distinguer les relevés de mêmes affinités en deux principaux groupes. Ceux situés à droite de l'axe 2 (G1) qui fournissent plus de quantité d'information des variables de forme et ceux se situant à gauche de cet axe (G2) qui en sont très pauvre. Les relevés $11 ; 12 ; 17$ et 18 de Diéfaye et ceux $1 ; 6$ et 37 de Cobitène sont plus riches en densité observée et en richesse spécifique moyenne. Par ailleurs, les relevé 1 et 37 de Cobitène sont plus riche en surface terrière et détiennent l'essentiel de l'information qui influence la corrélation notée.

\section{Les fonctions des vallées}

La répartition des citations sur les perceptions des populations quant aux fonctions que jouent les vallées de Ziguinchor montrent une différence significative au seuil
Alpha $(0,05)$ avec une probabilité de risque inférieure à $0,1 \%$ (Figure 6). L'analyse de cette figure montre que les vallées de Ziguinchor jouent essentiellement la fonction de production agricole selon $67,4 \%$ des citations. Les fonctions espace vert, réserve foncière, récréative et cultuelle occupent respectivement $12,3 \% ; 11 \% ; 7 \%$ et $2,2 \%$ de fréquence de citations. Les activités liées à la fonction de production agricole sont relatives à la riziculture sous pluie $(40,2 \%)$, au maraichage de contre saison $(38,1 \%)$ et au pâturage du bétail $(21,7 \%)$. La fonction de pâturage s'explique par le fait que les espèces herbivores (44,4\% composée de bovins; $13,8 \%$ de moutons; $13,2 \%$ de chèvres et $9,9 \%$ d'ânes) viennent paître l'herbe et les résidus rizicoles pendant la saison sèche. Il s'y ajoute les porcs $(15,9 \%)$ et les canards $(2,9 \%)$ qui y viennent chercher des vers de terre, des insectes; s'abreuver et/ou se rafraichir dans les points d'eaux.

La végétation ligneuse est délibérément laissée dans ces vallées, par les producteurs, en raison des différents services que les populations en tirent. A cet égard, la fonction de fertilisation des sols est la plus citée (28\%). Elle est suivie de l'ombrage, du bois d'énergie, du bois de service, des besoins cultuels qui concentrent respectivement $24,80 \% ; 23,00 \% ; 12,30 \%$ et $7,50 \%$ des citations. Les fonctions régulation du climat, de nichoirs d'oiseaux migrateurs et conservation de la biodiversité représentent $4,40 \%$ de citations. A propos de la fertilisation des sols, $48,40 \%$ des enquêtés s'accordent que les rendements rizicoles, évalué en moyenne à 4,79 t/ha, sont meilleurs sous couvert végétal dans les vallées. La majorité des enquêtés (99,3\%) accordent une importance particulière à l'espèce E. guineensis en raison de ses usages multiples. La différence de réapparition des opinions sur les usages de $E$. guineensis est significative au seuil Alpha 
$(0,05)$, avec une probabilité de risque inférieure à $0,01 \%$. La production des huiles de palme et de palmistes est l'usage le plus important de l'espèce ( $18,2 \%$ des citations). Il est suivi du vin de palme (17,9\%) et des lattes $(17,5 \%)$. La confection de balais avec les nervures principales des folioles $(15,6 \%)$, la construction de clôtures à l'aide de rameaux dans les blocs maraichers (15.2\%) et le bois de feux (11,5\%) justifient, par ailleurs, l'importance accordée au palmier à huile. Les autres usages $(4,1 \%)$ de cette espèce, dominante de la végétation ligneuse des vallées de la ville de Ziguinchor, s'articulent essentiellement autour de deux points. Il s'agit de ses vertus thérapeutiques dans la médecine traditionnelle et l'utilisation de la pulpe de noix de palme dans la préparation de la sauce appelé localement «Fiteuf» en Diola. Il résulte, d'ailleurs, de cette étude qu'un palmier à huile productif peut donner en moyenne 6,80 litres de vin de palme par jour pendant un mois dans l'année. Soit, une production d'environ, 204 1/palmier/an. Etant donné qu'un litre de vin de palme coûte $350 \mathrm{~F}$ $\mathrm{CA}$, un récolteur pourrait gagner environ 71 400 F CFA par pied/an. Considérant la densité moyenne des individus productifs d'un peuplement (52,19 pieds/ha), une vallée pourrait procurer un revenu monétaire considérable. Cependant, $87,60 \%$ des répondants reconnaissent que l'élagage des feuilles de E. guineensis contribue à la dégradation de la végétation des vallées de Ziguinchor.

Bien qu'ayant une fonction agricole et d'espace vert, les vallées de Ziguinchor subissent deux pressions majeures dues à l'occupation progressive par le bâti et la salinisation des sols. Ces facteurs contribuent considérablement à une perte des terres agricoles dans cette ville. D'ailleurs, 8,73\% des 838,9 ha que couvrent l'ensemble de ces vallées ne sont plus productifs, en termes de rendement rizicole, à cause de la salinité et de l'acidification des sols. Selon les enquêtés, bon nombre de projets de construction d'infrastructures dans ces vallées agricoles seraient sujets de litiges fonciers entre les autorités municipales et les producteurs. Il s'agit de la construction des zones d'habitats concertés, d'une usine de traitement des eaux usées et d'un camp de la marine dans la vallée de Cobitène. Il s'y ajoute la construction de la corniche routière qui passera par les vallées de Kenya, Kandialang et Djibock pour relier la ville au village de Boutoute. Par ailleurs, $88,9 \%$ des ménages agricoles de la ville s'aperçoivent que les déchets ménagers, en polyester, sont en train d'altérer la qualité des sols dans les vallées. Cet ensemble de facteurs justifient le pessimisme de $81,7 \%$ des enquêtés sur l'avenir des vallées et des activités agricoles dans la ville de Ziguinchor ; alors que $46,40 \%$ des ménages agricoles dépendent exclusivement de cette activité en termes de subsistance. D'ailleurs, les statistiques des enquêtes montrent que 13,1\% de ménages agricole de la ville de Ziguinchor sont des déplacés de la crise casamançaise, habitant les quartiers péri-urbains. Pourtant, en raison de leurs diverses fonctions environnementales et socioéconomiques, $74,5 \%$ des ménages optent pour la conservation de ces vallées en vue de promouvoir la sécurité alimentaire et le bienêtre des populations locales. 
Tableau 1 : Variation de la richesse spécifique dans les vallées de Ziguinchor.

\begin{tabular}{llllll}
\hline Variables & Diéfaye & Cobitène & Diéf+Cobit & p-value & Alpha \\
\hline R. spéci totale* & 68 & 67 & 90 & \\
R. spéci moy** & $10,94 \pm 3,82$ & $6,88 \pm 0,97$ & $8,26 \pm 1,36$ & 0,137 & 0,05 \\
Variance & 15,157 & 8,25 & 6,37 & \\
\hline *Richesse spécifique totale ; * Richesse spécifique moyenne ; Dief.=Diéfaye ; Cobit. =Cobitène ;
\end{tabular}

*Richesse spécifique totale ; ** Richesse spécifique moyenne ; Dief.=Diéfaye ; Cobit. =Cobitène ;

Tableau 2 : Variation de l'indice de diversité spécifique de Shannon.

\begin{tabular}{llll}
\hline & Cobitène & Diéfaye & Dief et Cobi \\
\hline $\mathrm{H}^{\prime}$ & $3,37 \pm 0,04$ & $2,93 \pm 0,08$ & $3,15 \pm 0,04$ \\
$\mathrm{Hmax}$ & $6,07 \pm 0,10$ & $6,09 \pm 0,30$ & $6,49 \pm 0,11$ \\
$\mathrm{R}$ & $0,56 \pm-0,09$ & $0,48 \pm 0,17$ & $0,49 \pm 0,07$ \\
\hline
\end{tabular}

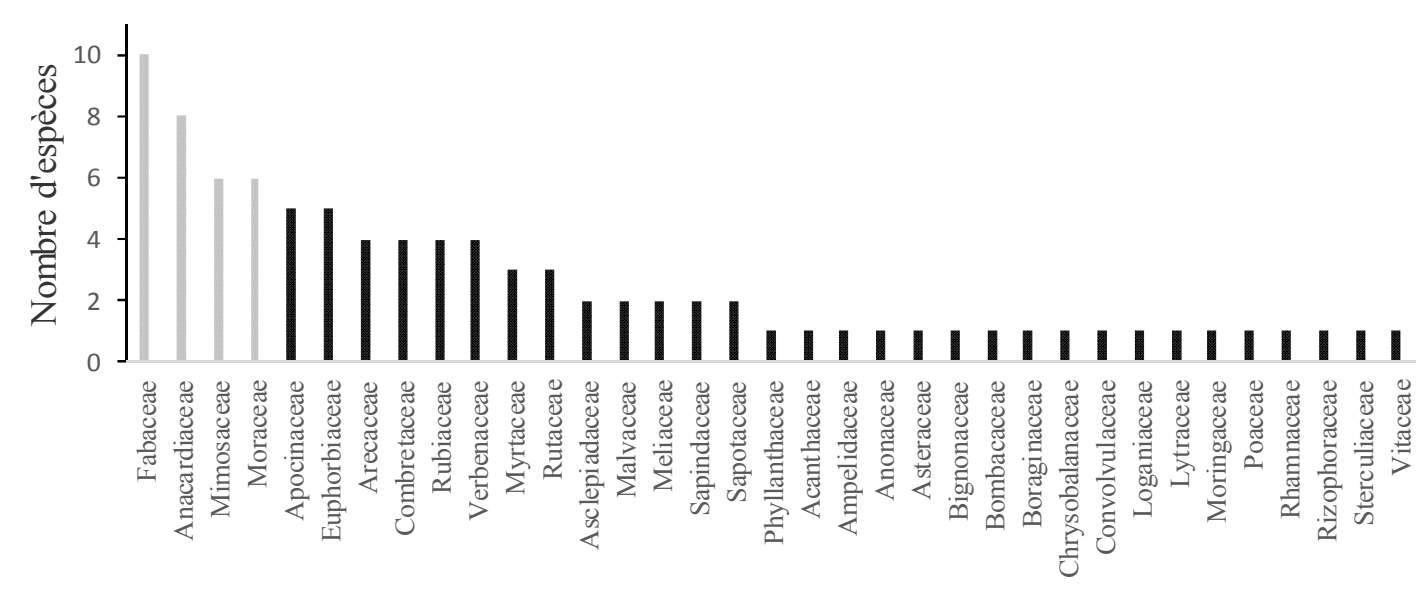

Familles botaniques

Figure2 : Répartition des espèces ligneuses dans les familles botaniques. 
Tableau 3 : Répartition des espèces dans les vallées par classes de fréquence.

\begin{tabular}{|c|c|c|c|c|}
\hline \multirow{2}{*}{ Fréq. de présence } & \multicolumn{2}{|c|}{ Nombre d'espèces } & \multirow{2}{*}{ Indice } & \multirow{2}{*}{ Quantification } \\
\hline & Diéfaye & Cobitène & & \\
\hline$] 0 ; 0,2[$ & 48 & 62 & I & Rares \\
\hline$[0,2 ; 0,4[$ & 13 & 3 & II & Accessoires \\
\hline$[0,4 ; 0,6[$ & 4 & 1 & III & Fréquente \\
\hline$[0,6 ; 0,8[$ & 2 & 1 & IV & Abondantes \\
\hline$[0,8 ; 1[$ & 1 & 0 & $\mathrm{~V}$ & Constantes \\
\hline
\end{tabular}

Tableau 4 : Variation des taux de régénération, d'anthropisation et de mortalité de la flore.

\begin{tabular}{|c|c|c|c|c|c|c|}
\hline Variables & Cobitène & & Diéfaye & Dief. et Cobi. & Alpha & p-value \\
\hline Tx.RN(Toltal) (\%) & $60,01 \pm 6,90$ & 10,90 & 63,31 & $61,14 \pm 5,41$ & 0,05 & 0,4 \\
\hline ISR (E. guin) $(\%$ & $23,69 \pm 8,72$ & 11,44 & 51,95 & $33,30 \pm 7,16$ & & \\
\hline Tx.Anthrop (\%) & $35,84 \pm 7,08$ & 10,73 & 33,23 & $34,95 \pm 5,47$ & & \\
\hline Tx.mort (\%) & $6,13 \pm 3,04$ & 1,19 & 3,94 & $5,39 \pm 1,93$ & & \\
\hline
\end{tabular}

Tx.RN : Taux de régénération naturel; ISR (E. guin) : Importance spécifique de régénération de Elaeis guineensis ; Tx.mort:

Taux de mortalité ; Tx.Anthrop : Taux d'anthropisation.

Tableau 5 : Paramètres structuraux de la végétation ligneuse des vallées de Ziguinchor.

\begin{tabular}{|c|c|c|c|c|c|}
\hline & Cobitène & Diéfaye & Dief et Cobi & p-value & Alpha \\
\hline D.obs Total (ha) & $136,90 \pm 43,26$ & $393,78 \pm 148,09$ & $216,62 \pm 63,84$ & 0,191 & 0,05 \\
\hline D.obs E.guin(ha) & $53,40 \pm 15,89$ & $266,22 \pm 118,71$ & $119,45 \pm 46,80$ & & \\
\hline Hmoy (m) & $7,85 \pm 0,47$ & $9,91 \pm 0,67$ & $8,88 \pm 0,57$ & & \\
\hline $\mathrm{C}\left(\mathrm{m}^{2} \cdot \mathrm{ha}^{-1}\right)$ & $862,59 \pm 270,27$ & $1976,08 \pm 1118,80$ & $1208,15 \pm 423,12$ & & \\
\hline $\mathrm{St}\left(\mathrm{m}^{2} \cdot \mathrm{ha}^{-1}\right)$ & $5,49 \pm 2,18$ & $8,34 \pm 3,68$ & $6,37 \pm 1,95$ & & \\
\hline
\end{tabular}

D.obs : Densité observée; E.guin: Elaeis guineensis ; Hmoy: Hauteur moyenne; $\mathrm{C}\left(\mathrm{m}^{2} \cdot \mathrm{ha}^{-1}\right)$ : couvert ligneux ; $\mathrm{St}\left(\mathrm{m}^{2} \cdot \mathrm{ha}^{-1}\right)$ : Surface terrière. 


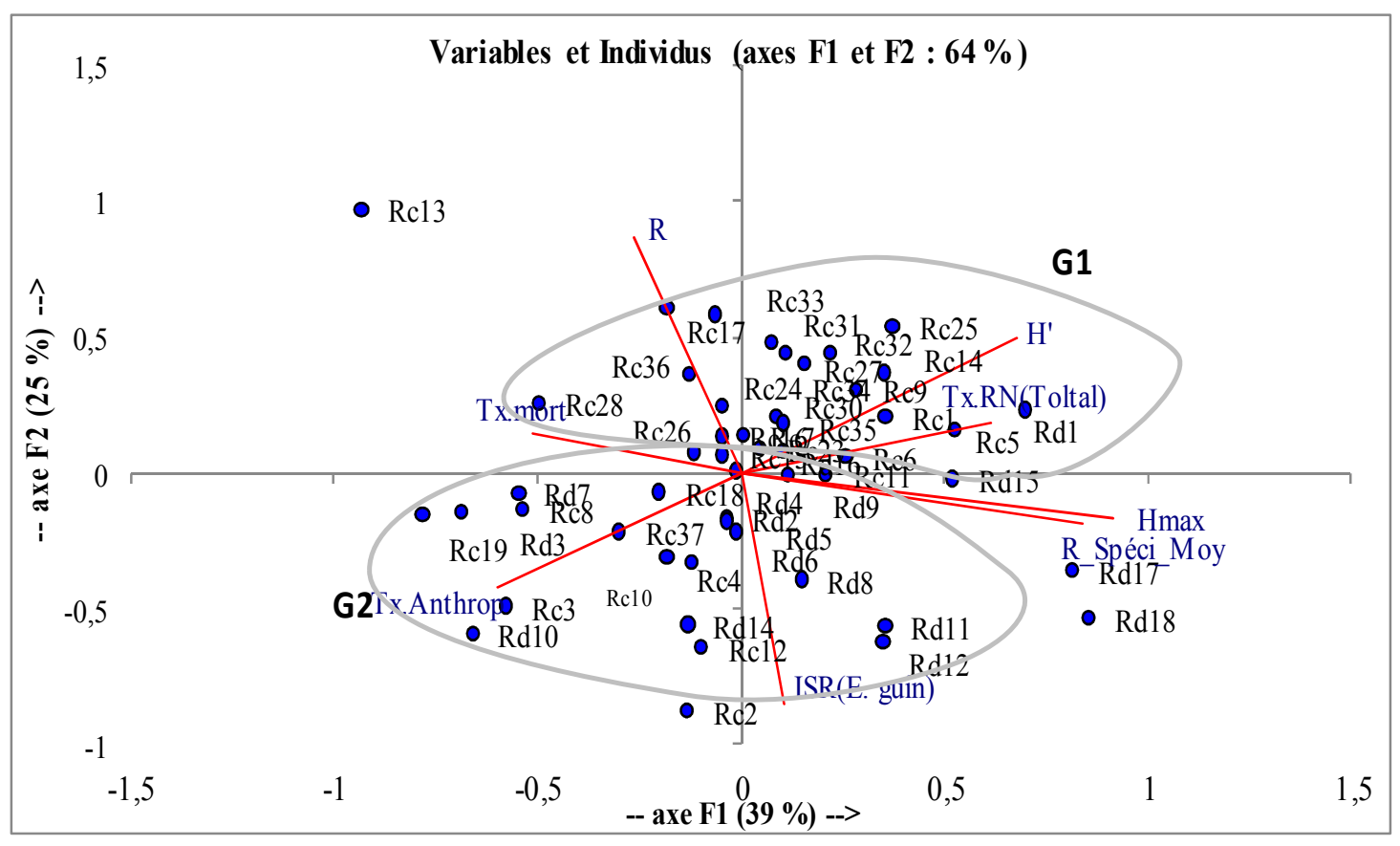

Figure 3: Positionnement des variables R_Spéc_moy, Tx.RN, ISR(E.guin), Tx.mort, Tx. Anthrop, H', Hmax, $\mathrm{R}$ et des relevés de végétation des deux vallées dans un système d'axe (Analyse à Composante Principale).

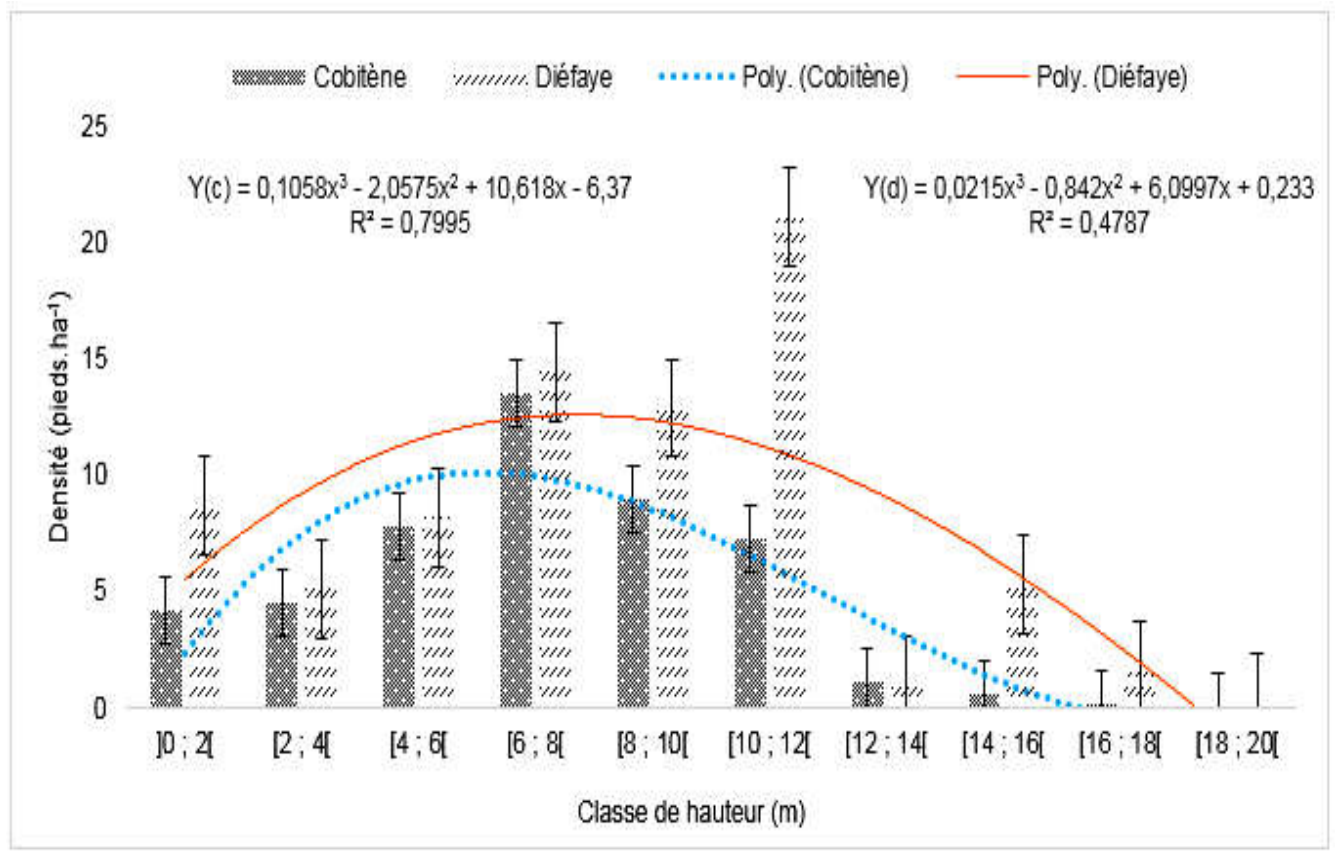

Figure 4: Structure verticale du palmier à huile dans les vallées de Diéfaye et Cobitène. 


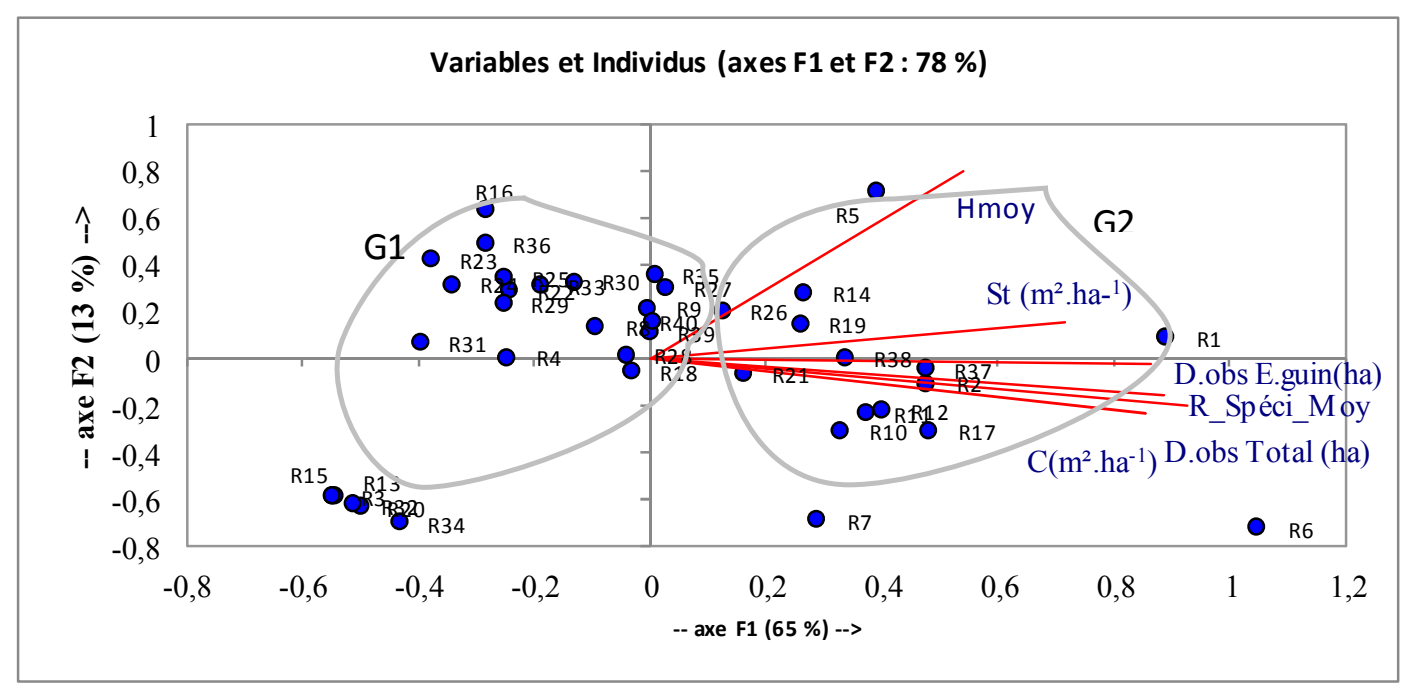

Figure 5: Distribution des paramètres de formes du peuplement dans le plan factoriel F1\&F2.

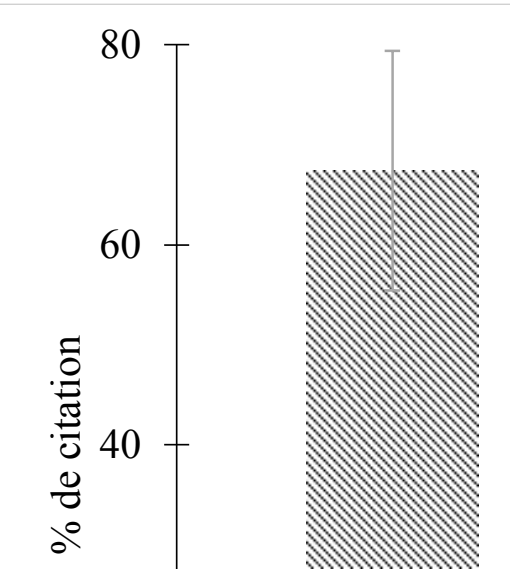

Figure 6: Fonctions reconnues des vallées de Ziguinchor.

\section{DISCUSSION}

La discussion des résultats porte principalement sur la flore, les usages post conflit, le rôle environnemental et socioéconomique des vallées de la ville de Ziguinchor et les enjeux de l'occupation du sol par le bâti.

\section{Sur la Diversité floristique des vallées}

La richesse spécifique totale de la flore ligneuse des vallées de la ville de Ziguinchor est relativement élevée (90 espèces réparties dans 79 genres et 35 familles). Cette diversité de la flore ligneuse des vallées agricoles de Ziguinchor est plus importante que celle évaluée à 63 espèces ligneuses réparties en 52 genres, relevant de 24 familles dans les parcs agroforestiers à E. guineensis des villages de la basse Casamance par Gomis (2016). L'indice de diversité spécifique de Shannon estimée 3,15 bits est plus importante que celui estimé à 3,09 bits par Diédhiou et al. (2014) dans les parcs agroforestiers à Faidherbia albida (Del.) Chev. dans le terroir insulaire de 
Mar Fafaco. Ces résultats montrent que les zones de culture de la ville de Ziguinchor sont plus riches en, termes de biodiversité, que celles des terroirs villageois de la basse Casamance et du bassin arachidier au Sénégal. Ce qui corrobore les conclusions de Montiel (2015) ; qui avait, déjà, confirmé l'hypothèse selon laquelle l'agriculture urbaine contribue plus à la conservation de la diversité biologique que celle pratiquée en campagne.

\section{Sur la comparaison de la flore et de la végétation des vallées}

L'indice de dissimilarité de Jaccard déterminé entre les vallées de Diéfaye et Cobitène est évalué à 0,53 . Il est proche de sa valeur médiane et traduit ainsi la relative similarité, en termes de richesse spécifique, entre les deux vallées. Cependant, la variance de la richesse spécifique moyenne de la vallée de Diéfaye représente, environ, le double de celle de Cobitène. Ce qui montre que le peuplement de Cobitène est relativement plus homogène que celui de Diéfaye. L'espèce $E$. guineensis est la plus dominante, aussi bien à Diéfaye qu'à Cobitène, avec une fréquence de présence d'environ $70 \%$. On peut donc noter que ces vallées, généralement destinées à la riziculture pluviale et au maraichage de contre saison, constituent des parcs à E. guineensis. L'importance spécifique de régénération de cette espèce dans la vallée de Diéfaye représente deux fois celle de Cobitène. Ce qui montre que le peuplement du palmier à huile se régénère plus à Diéfaye qu'à Cobitène. Cet état des faits pourrait être lié à l'intensité des activités anthropiques qui est plus élevée à Cobitène qu'à Diéfaye. Les activités anthropiques inhibant la régénération naturelle sont liées aux brulis des résidus agricoles, à l'élagage de la palmeraie pour la construction des clôtures et lutter contre la dent du bétail en divagation dans les espaces maraichers. C'est ce qui serait responsable, en partie, de la mortalité et de la baisse de productivité du palmier à huile comme l'atteste $87,60 \%$ des enquêtés. A ce propos, la totalité des enquêtés, ayant plus de 50 ans, sont d'avis que la pression anthropique exercée sur la végétation de la ville de Ziguinchor serait responsable de la baisse considérable de sa densité, comparée à son état il y'a 30 ans. D'ailleurs, les coefficients de corrélation entre le taux d'anthropisation et la richesse spécifique moyenne, d'une part; et, le taux d'anthropisation et le taux régénération naturelle, d'autre part, sont négatifs et relativement élevés en valeurs absolues. Ils corroborent ainsi que l'anthropisation a des effets négatifs sur la régénération naturelle et la diversité spécifique de la végétation des vallées de Ziguinchor. On note que 63,29\% des individus de E. guineensis sont principalement concentrés dans les classes de hauteurs comprises entre 8 et $12 \mathrm{~m}$ à l'échelle des deux vallées. Par ailleurs, la hauteur moyenne du peuplement est de 8,88 $\pm 0,57 \mathrm{~m}$. Ces résultats révèlent le caractère arborescente de la végétation ligneuse de la ville de Ziguinchor. Les relevés 1 et 37 de Cobitène fournissent plus de quantité d'informations en termes de surface terrière. Cette particularité est liée à la présence des sujets de Ceiba pentandra (L.) Gaertn. et Adansonia digitata L. présentant de gros troncs. Ces lieux constituent d'ailleurs des sites de culte sacrés des femmes des quartiers de Cobitène et Colobane.

\section{Sur les usages post-conflit des vallées}

A la suite de l'accroissement de la population et de leurs besoins alimentaires, l'occupation des sols de Ziguinchor s'est traduite par une forte pression sur les terres des vallées. A cet égard, les vallées ont toujours joué une fonction de production agricole dans la ville. Parmi les formes d'accès à la terre, la location et l'emprunt $(60,64 \%$ des cas) démontrent la pression foncière sur les ressources pédologiques à la 
suite du conflit casamançais (Dasylva, 2013). D'ailleurs Robin et Ndione (2006) ont démontré que l'installation des populations des arrondissements de Nyassia et Niaguis dans les quartiers péri-urbains de Ziguinchor, à la suite du conflit casamançais, a accru la pression démographique sur ses terres. Cette donne a entrainé des mutations de la tenure foncière dominée par l'emprunt et la location aux dépens de l'héritage familial jadis ancré en Casamance. Cette fonction des vallées contribue à la sécurité alimentaire des populations autochtones et des déplacées, grâce à la production rizicole autoconsommée. Cette autoconsommation assure plus de 12 mois de subsistance à $12 \%$ des ménages qui ont des exploitations agricoles dans les vallées. Cette assertion est confortée par l'importance des rendements moyens de ce type de production évalué à 4,79 tonnes de riz à l'hectare. Par ailleurs, la production maraichère contribue aussi à la lutte contre la pauvreté grâce aux revenus moyens par producteur évalués à $485000 \mathrm{~F}$ CFA par Dasylva (2013). Il s'y ajoute que ces vallées jouent le rôle de fonctions de pâturage et d'abreuvoir du bétail. Au regard de ces constats, tout programme de réinsertion des personnes déplacées doit être réfléchi, a fortiori lorsqu'il s'agit d'une population aussi cosmopolite que celle de la région de Ziguinchor (Robin et Ndione, 2006). En outre, les populations de Ziguinchor perçoivent bien l'importance agro-écologique de l'arbre dans les bas-fonds. Cette étude a, d'ailleurs, montré que la fertilisation des sols est l'une des raisons fondamentales de la présence des arbres dans les vallées.

\section{Sur les rôles environnementaux des formations végétales des vallées}

La régulation du climat par les arbres en zone urbaine est moins perçue par les enquêtés (12,30\%), comparée à la fertilisation des sols (28\%). Pourtant, les travaux de
Johnston et Newton (2004) ont montré qu'en zone urbaine, un arbre mature transpirant 450 litres d'eau, a un effet refroidissant équivalent à celui de 5 climatiseurs fonctionnant 20 heures par jour. La végétation de ces vallées contribue donc à la réduction de certains maux urbains; comme la pollution de l'eau et de l'air ou l'îlot de chaleur urbain et rendent plus attractive la ville comme le montrent les travaux de Kervadec (2011) et Long et Tonini (2012). E. guineensis fournit de multiples services aux populations. Ils sont essentiellement liés à la production des huiles utilisées dans l'assaisonnement des mets. Il s'y ajoute la récolte du vin de palme, utilisé lors des rites sacrés des communautés Diola, Manjack et Mankagne. Ces groupes ethniques, fortement rattachés à leurs coutumes et à l'agriculture, constituent respectivement, $75,80 \% ; 2,60 \%$ et $0,70 \%$ des ménages enquêtés. La valeur monétaire annuelle d'un palmier à huile, productif en termes de vin de palme, est estimée à 71400 CFA ce qui confirme son importance socioéconomique.

\section{Sur les enjeux de l'occupation des vallées par le bâti}

$\mathrm{Si}$ les autorités municipale ne perçoivent que la fonction réserve foncière de ces vallées pour l'extension de la ville, ils ne doivent pas perdre de vue qu'elles sont non aedificandi et caractérisées par une nappe phréatique peu profonde. Leur occupation par les habitats, exposerait les populations à des inondations récurrentes et à l'abandon de l'agriculture. D'ailleurs Sy et Sané (2011) ont déjà notés des inondations dans le quartier de Goumel sis dans des casiers rizicoles remblayés. Selon le GFDRR (2014), plus de 70 milliards de FCFA, compte non tenu des appuis extérieurs, ont été injectés par l'Etat du Sénégal dans la lutte contre les inondations, entre 2008 et 2012 avec des résultats mitigés. Ce même phénomène a concerné 250.000 familles dans les zones périurbaines de Dakar 
la même année (ANSD, 2010). La présence des vallées, exutoires des eaux de ruissellement, explique leur rôle d'atténuation des inondations notées à Ziguinchor. Cependant, les autorités municipales ne doivent pas faire abstraction du caractère non aedificandi des vallées pour en faire une zone d'habitats. D'ailleurs, face à l'option de juguler la croissance urbaine par les terres des vallées, $74,5 \%$ des ménages plaident pour la sauvegarde de leurs valeurs agro écologiques.

\section{Conclusion}

Cette étude montre les caractéristiques de la végétation ligneuse des vallées de Ziguinchor et indique les fonctions essentielles que les populations locales leur attribuent. La flore ligneuse de ces vallées compte 90 espèces réparties dans 79 genres et 35 familles botaniques. La famille des Fabaceae, des Anacardiaceae, respectivement composées de 10 et 7 espèces chacun, sont les plus représentés. L'indice de dissimilarité de Jaccard $(0,53)$ est proche de sa valeur médiane et traduit la relative similarité de la flore ligneuse des vallées de Cobitène et Diéfaye. L'indice de diversité de Shannon est relativement élevé (3,15 bits) dans les deux vallées. Il est cependant plus important dans la vallée de Cobitène que celle de Diéfaye. Les vallées de Ziguinchor peuvent être qualifiées de parc agroforestier à Elaeis guineensis en raison de la dominance de l'espèce avec une fréquence de présence de plus de $70 \%$. Il s'y ajoute que ces vallées jouent principalement la fonction de production agricole selon $67,40 \%$ des citations. Les systèmes de production agricoles qui y sont pratiquées sont liées à la riziculture pluviale caractérisée par des rendements de 4,79 t/ha, au maraichage de contre saison et au pâturage du bétail. Face à l'ignorance des fonctions des vallées au profit de la croissance urbaine, $74,5 \%$ des ménages plaident pour le maintien de leurs valeurs agroécologiques. En perspective, la fonction de réserve foncière dévolue aux vallées par les autorités municipales doit être abandonnée pour asseoir la sécurité alimentaire et le bienêtre des populations à la périphérie de Ziguinchor.

\section{REMERCIEMENTS}

Les auteurs remercient les doctorants de la première promotion du Laboratoire d'Agroforesterie et d'Ecologie de l'Université Assane Séck de Ziguinchor, pour leur vaillante participation à la collecte des données ayant permis la rédaction de l'article. Nous remercions aussi 1'Université de LAVAL, qui à travers son $2^{\circ}$ Colloque « Etudiant en développement international », a suscité l'intérêt et le choix du sujet de thèse de Maurice DASYLVA portant sur l'agriculture urbaine à Ziguinchor. Mention spéciale aux chercheurs de l'UMR Innovation de SupAgro de Montpelier, particulièrement à Christophe SOULARD, pour m'avoir accueilli en stage de recherche en 2014 et pour l'intérêt manifeste qu'il accorde à l'encadrement de mes travaux de thèse.

\section{CONFLIT D'INTERETS}

Les auteurs ci-après déclarent, à l'unanimité, qu'il n'y a aucun conflit d'intérêts qui serait liée au présent article.

\section{CONTRIBUTION DES AUTEURS}

MD: Elaboration de l'approche méthodologique de recherche, inventaire de la végétation ligneuse des vallées agricoles, réalisation des enquêtes socioéconomiques et ethnobotaniques, traitement et analyse des données et rédaction de l'article. NN: Cosuperviseur des travaux, contribution à la rédaction de l'approche méthodologique de recherche, à la collecte et analyse des données et la rédaction de l'article. ON: Collaborateur dans la collecte et le traitement statistique des données, contribution dans la rédaction de l'article. BS: superviseur des travaux, 
contribution au choix et à la définition du sujet, orientation des travaux de recherche, contribution à la rédaction de la méthodologie de recherche.

\section{REFERENCES}

Akpo LE, Grouzis M. 1996. Influence du couvert sur la régénération de quelques espèces ligneuses sahéliennes (NordSénégal, Afrique occidentale). Webbia, 50(2): 247-263.

ANSD (Agence Nationale des Statistiques Démographiques). 2010. Situation économique du Sénégal en 2009. Dakar, Sénégal, ISSN 0850-1491, 19 P.

ANSD. 2014. Recensement Général de la Population et de l'Habitat, de l'Agriculture et de l'Elevage (RGPHAE) 2013, Rapport provisoire, 36 P.

ANSD. 2015. Rapport de projection de la population du Sénégal: 2013-2063, 175 P.

Arbonnier M. 2009. Arbres, arbustes et lianes des zones sèches de l'Afrique de l'Ouest, Ed. Quae, Paris, ISBN MNHN 978-2-85653-626-1, $577 \mathrm{p}$.

Berhaut J, 1967. Flore du Sénégal (2 $2^{\mathrm{ème}}$ Edn). Clairafrique : Dakar ; 485 p.

Bonnet P, Arbonnier M, Grard P. 2005. Ligneux du Sahel. - CIRAD - CD-Rom.

Boucher I, Fontaine N. 2010. La biodiversité et l'urbanisation, Guide de bonnes pratiques sur la planification territoriale et le développement durable, ministère des Affaires municipales, des Régions et de l'Occupation du territoire, coll. «Planification territoriale et développement durable», ISBN 978-2550-60459-4, $\quad 178$ p. [www.mamrot.gouv.qc.ca].

Dasylva M. 2013. Agriculture urbaine sous pluie et sécurité alimentaire dans la commune de Ziguinchor. Mémoire de Master, Département d'agroforesterie, UFR-ST, UASZ, 51p.
Diédhiou MAA, Faye E, Ngom D, Touré MA. 2014. Identification et caractérisation floristiques des parcs agroforestiers du terroir insulaire de Mar Fafaco, Sénégal. Journal of Applied Biosciences, 79: 6855-6866 ; ISSN 1997-5902.

Diané SL. 2000. Approches biogéographiques de la nature en ville. Parcs, espaces verts et friches». Cahiers de Géographie du Québec, 44(122): 147166.

Faye E. 2005. Étude floristique, phytosociologie, phytogéographique, ethnobotanique, et de la régénération après coupe des ressources ligneuses dans la région de Kaolack (Sénégal). DEA Université Libre de Bruxelles, 83p.

Faye E. 2010. Diagnostic partiel de la flore et de la végétation des Niayes et du Bassin arachidier au Sénégal: application de méthodes floristique, phytosociologique, ethnobotanique et cartographique. Thèse de Doctorat, Université Libre de Bruxelles, 253p.

Frontier S, Pichod-Viale D. 1995. Écosystèmes : $\quad$ Structure, Fonctionnement, Evolution. Masson: $447 \mathrm{p}$.

GFPRR (Global Facility for Disaster Redaction and Recovery). 2014. Sénégal: Inondations urbaines, Le Relèvement et la Reconstruction à partir de 2009. Rapport de consultation, $27 \mathrm{p}$.

Gomis ZD. 2016. Les parcs agroforestiers à Elaeis guineensis Jack. (Palmier à huile): Caractéristiques biophysiques et importance socio-économique en BasseCasamance. Mémoire de Master, Département d'agroforesterie, UFR-ST, UASZ, 74p.

Johnston J, Newton J. 2004. Building Green: A guide to using plants on roofs, walls and pavements, Greater London Authority. London ISBN: 1852616377. 
Keita A. 2013. La mutation des terres agricoles autour de Ziguinchor; Mémoire de master ATDDL, FLSH, UCAD, 96p.

Kervadec T. 2011. Intégrer la nature en ville dans le Plan local d'urbanisme : Observation, analyse, recommandations, MEDTL, Etd/France, www.projetdeterritoire.com , $44 \mathrm{p}$.

Long N, Tonini B. 2012. Les espaces verts urbains : étude exploratoire des pratiques et du ressenti des usagers. Natures et Métropoles, 12(2).

Mbaye I, Sané T, Diéye EB. 2011. Changement climatique, perceptions et stratégies des populations de la ville de Ziguinchor au Sénégal; Actes du colloque "Renforcer la résilience au changement climatique des villes: du diagnostic spatialisé aux mesures d'adaptation" (2R2CV) 07 et 08 juillet 2011, Université Paul Verlaine-Metz, France; $9 \mathrm{p}$.

Montiel MS. 2015. Le Programme d'Agriculture Urbaine de la ville de Rosario en Argentine ", Revue d'Ethnoécologie [En ligne], URL : http://ethnoecologie.revues.org/2390 ; DOI : 10.4000/ethnoecologie.2390.

Ngom D. 2008. Définition d'indicateurs de gestion durable des ressources sylvopastorales au Ferlo (Nord-Sénégal), Thèse de Doctorat en Biologie végétale, Option : Écologie, FST, UCAD, 148 p.

Oladokoun W. 2013. L'inefficacité du modèle de gestion du foncier rural au Togo. Etude de cas des agglomérations rurales périurbaines d'Atakpamé. Revue de Géographie Tropicale et d'Environnement, 1: 45-65.

PAM (Programme Alimentaire Mondial). 2012. Rapport d'évaluation de la sécurité alimentaire en milieu urbain; communes de Kaolack, Kolda, Tambacounda et Ziguinchor; Rapport national du Sénégal; 24p.

Piélou EC. 1966. The measurement of diversity in different types of biological collections. Journal of Theoretical Biology, 13: 131-144.

Poupon H. 1980. Structure et Dynamique de la Strate Ligneuse d'une Steppe Sahélienne au Nord du Sénégal. ORSTOM éd. (Etudes \& Thèses): Paris ; $307 \mathrm{p}$.

Ramade F. 2003. Eléments d'Ecologie, Ecologie Fondamentale. Ed. Dunod: Paris ; 690p.

Robin N, Ndione B. 2006. L'accès au foncier en Casamance. L'enjeu d'une paix durable? Handicap international, Dakar, 15 P.

Sagna P. 2005. Dynamique du climat et son évolution récente dans la partie ouest de l'Afrique occidentale, Thèse de doctorat d'Etat ES Lettre, UCAD, Faculté des lettre et Sciences Humaine, Département de Géographie, 790p.

Sy O, Sané T. 2011. Périurbanisation et vulnérabilité dans la ville de Ziguinchor (Sénégal) : le cas du quartier Goumel. Colloque International sur " Aménagement périurbain : processus, enjeux, risques et perspectives ». Laboratoire d'Analyse GéoEnvironnementale et d'Aménagement (LAGEA) de la faculté des Lettres et des Sciences Humaines Sais, Fès ; 139-151. 